\title{
Trans-Jacket Fibre Bragg Gratings for In-Situ Health Monitoring of Defence Platforms in Harsh Environments
}

\author{
Naizhong Zhang ${ }^{1, a^{*}}$, Claire Davis ${ }^{2, b}$, Chiu Wing Kong ${ }^{1, c}$, Suzana Turk ${ }^{2, d}$ \\ 1Department of Mechanical and Aerospace Engineering, Monash University, Wellington Rd, \\ Clayton VIC. 3800, Australia \\ 2Defence Science and Technology Group, 506 Lorimer Street, Fishermans Bend, VIC 3207, \\ Australia \\ aNaizhong.Zhang1@monash.edu, bClaire.Davis@dst.defence.gov.au, \\ cWing.Kong.Chiu@monash.edu, dSuzana.Turk@dst.defence.gov.au
}

\begin{abstract}
Keywords: Optical Fibre Sensors, Fibre Bragg Gratings, Grating Inscription, TransJacket Gratings, Sensor Fatigue
\end{abstract}

\begin{abstract}
Packaged optical fibre sensors offer excellent strength and resistance to environmental degradation, but the reported reliability and durability of fibres containing fibre Bragg gratings (FBGs) varies greatly. This is partly due to the fabrication methodologies used to create the sensors. The trans-jacket grating inscription technique uses an infrared laser to write gratings into the fibre core through the polymer coating. This method eliminates the need for harsh coating removal processes and exposure of the glass fibre core and thus dramatically reduces fibre damage during grating fabrication. In addition, the automated trans-jacket inscription process introduces greater flexibility to control the writing parameters, facilitating a consistent process for producing robust, fatigue resistant distributed FBG sensing arrays with reliable and repeatable performance that could revolutionise their application in structural health monitoring (SHM). This paper reports on the durability and reliability of Bragg gratings with different fibre geometries, dopants, and photo-sensitising approaches to compare the overall fatigue performance of trans-jacket FBG sensors. Both type I gratings which are inscribed using a laser power intensity below the damage threshold of the glass core, and type II gratings which are inscribed exceeding this threshold, are considered. The fatigue performance of these FBG sensors was assessed using a custom designed electro-dynamically actuated loading assembly. It is concluded that type I trans-jacket gratings have a significantly higher fatigue life compared to type II gratings for the same fatigue loading regime. Despite the lower fatigue life, type II transjacket gratings are found to perform significantly better than conventional electrical foil gauges. Therefore, trans-jacket gratings have significant potential for application as dense sensing arrays in harsh operational environments in defence and aerospace industries.
\end{abstract}

\section{Introduction}

FBGs are periodic variations in the refractive index of the fibre core. They are inscribed by photo-sensitising the glass and then exposing the fibre core side to laser light with a spatially modulated intensity pattern [1,2]. The grating is designed to act as a narrow-band reflector, reflecting light of a specific wavelength known as the Bragg wavelength and transmitting light at all other wavelengths. FBG sensors have been widely used as sensing elements for strain, temperature, and pressure measurement over the last decade [3-5].

FBG sensors are small, flexible, relatively simple to fabricate, corrosion resistant, and immune to electromagnetic interference (EMI), making them well suited to embedded strain and 
temperature monitoring applications [6-8]. However, the reported performance variability of FBG sensors impedes their wider acceptance and application. This is partly due to the various commercial fabrication methodologies used to create the sensors [9]. For example, the conventional stripped and recoated FBG writing method involves manual handling that introduces surface damage in the unprotected region of the glass, which affects the fibre's longterm reliability and durability [10,11]. Ang et al [12] reported a fatigue failure strain of $5000 \mu \varepsilon$ when a stripped and re-coated FBG embedded in composite material was tested, which is only $10 \%$ of the tensile strain limit for pristine fibres.

Trans-jacket inscription of FBGs relies on femtosecond laser pulses to direct focused energy into the fibre core through the fibre coating. This technique relies on maintaining the laser focus in the core of the fibre to avoid damage to surrounding fibre and coating materials. This is a relatively new technique which has emerged following recent improvements in beam stability and pulse energy for ultrafast lasers. The resulting gratings maintain the mechanical robustness of the pristine fibre, which is paramount for fatigue resistance [13]. A novel aspect to the inscription methodology employed by these researchers was an active feedback mechanism to keep the beam focused in the core of the fibre during inscription, which may be a factor in the superior fatigue performance of these gratings. This methodology was employed to manufacture both type I and type II FBGs in the current study, providing a set of sensors for systematic fatigue testing.

FBGs written using continuous wave $(\mathrm{CW})$ lasers at low energy intensities are referred to as type I gratings, also known as standard gratings [2]. In the present work, the type I trans-jacket FBGs were manufactured according to the experimental procedure and parameters detailed in $[13,14]$. Type II gratings, also called damage induced gratings, are written by multi-photon excitation with higher intensity laser energies that exceed the damage threshold of the glass core [2]. Type II gratings can tolerate much higher temperatures compared with type I gratings, and are often adopted in high temperature sensing environments. The fatigue performance of type II gratings has not been extensively studied but, given that the glass is essentially damaged in the region of the grating $[15,16]$, it is expected that there will be some deterioration in the fatigue performance compared to type I gratings.

The fatigue performance characteristics of foil strain gauges (FSG) are not ideal for long-term full-scale fatigue testing (FSFT) of aerospace platforms [17]. In a previous fatigue study FSGs tested at a load amplitude of $1000 \mu \varepsilon$ were found to last in the order of 100 million cycles; at $5200 \mu \varepsilon$ the fatigue life was significantly less, $\sim 1000$ cycles, and at $7000 \mu \varepsilon$ the fatigue life was further reduced to $\sim 100$ cycles [18]. It was considered unusual for a typical FSG to survive more than 200,000 cycles at $4000 \pm 2000 \mu \varepsilon$ [19]. Thus, the prospect of a durable and fatigue-resistant optical fibre strain sensor offers significant benefits for this and similar applications. The wiring and soldered connections associated with a large number of foil strain gauges can lead to reliability issues and excessive weight on the structure can influence test results. In addition, the ability to multiplex large numbers of sensors along a single fibre enables significant scale and complexity reduction relative to a wired sensor system, using a medium which is immune to electromagnetic interference. To validate that trans-jacket FBGs have potential to replace FSGs in the field for long-term structural health monitoring of aerospace platforms, this paper evaluates the fatigue performance of type I and type II trans-jacket FBG sensors fabricated with different fibre geometries, dopants, and photo-sensitising approaches. 


\section{Experimental Methods}

A novel electro-dynamically actuated fibre loading rig was designed and constructed which can operate at relatively high frequency (see Fig. 1). The dynamic range of this rig was designed to induce tensile strains in an optical fibre of up to $36,000 \mu \varepsilon$ at a fixed $100 \mathrm{~Hz}$ cyclic loading frequency [20].

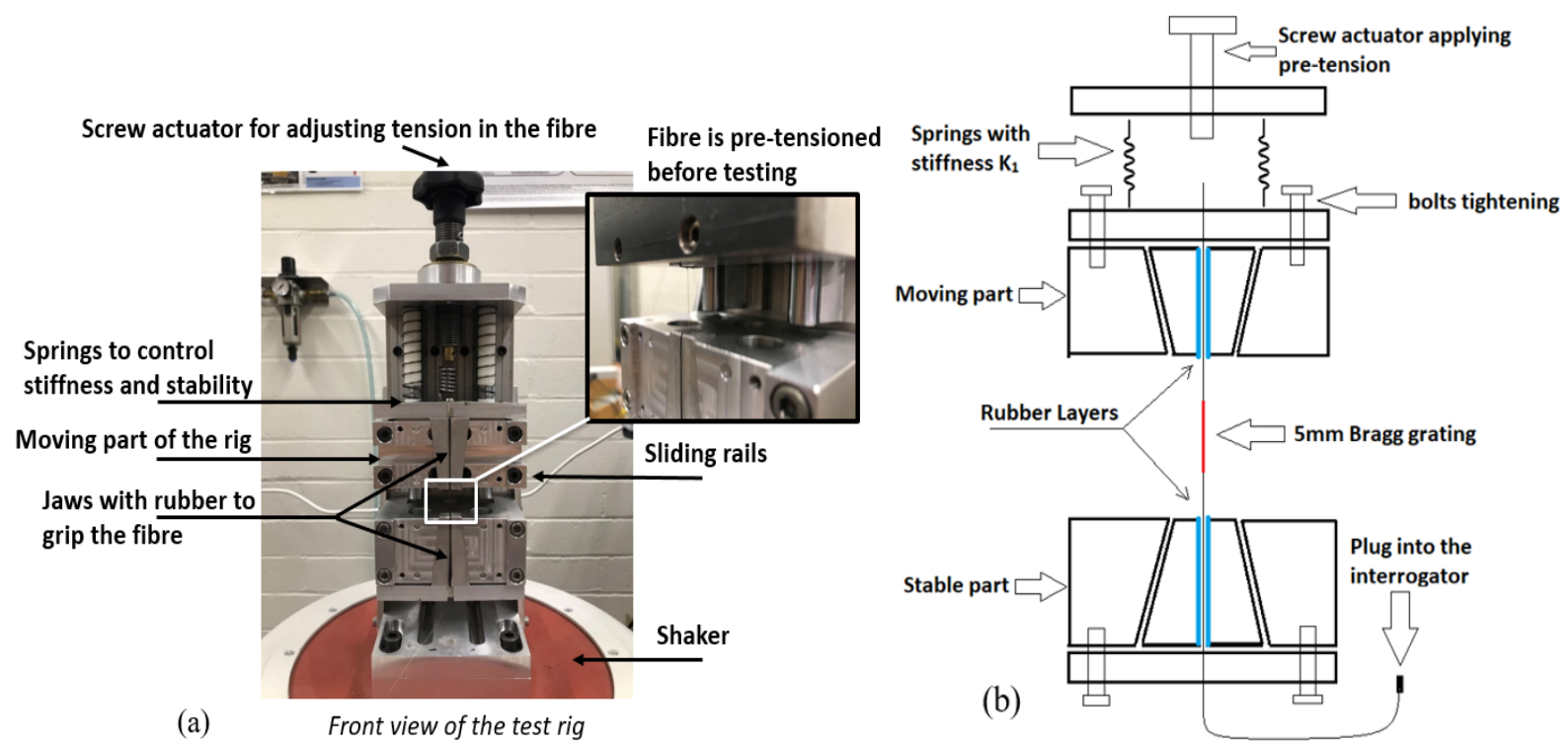

Figure 1. (a) Picture of optical fibre test rig bolted to an electrodynamic shaker. (b) Schematic illustrating working principle of the test rig [20], with the FBG mounted between the moving part and the stable part (labelled).

The reflection and transmission spectra are mainly determined by the grating length, index contrast, and grating pitch of the FBG sensor [21]. Pre- and post-fatigue test FBG reflection and transmission spectra were characterised by a high-resolution tunable laser (Yenista TS100) combined with a component tester (Yenista, CT400). The key features of the FBGs, such as centre Bragg wavelength, side lobes, and reflectivity were studied to determine whether fatigue loading had any impact on the spectral profile and, in the case of the fractured fibres, whether the fracture occurred within the region of the grating, which is indicated by a drop in reflectivity.

Constant amplitude sinusoidal loading was delivered by a high capacity electrodynamic shaker (TIRA S50350) controlled by a Vibration Research Corporation 8500 vibration controller. The amplitude of this loading was adjusted manually to achieve the desired level of cyclic strain in the fibre using the fibre signal as feedback, see Fig. 2 (a). Fig. 2 (b) shows a signal from a trans-jacket FBG sensor corresponding to a strain excursion from 0 to $25,000 \mu \varepsilon$ at $100 \mathrm{~Hz}$.

The strain induced in the FBG sensor was measured continuously using an industrial grade optical sensing interrogator (Micron Optics Si255). The Bragg wavelength for an unstrained FBG sensor tested in the loading rig was $1560 \mathrm{~nm}$. The available dynamic strain range of the interrogator is approximately $130,000 \mu \varepsilon$ across a wavelength of $1460 \mathrm{~nm}$ to $1620 \mathrm{~nm}$. Therefore, the interrogator provides sufficient wavelength range for the strains considered in the present work. 

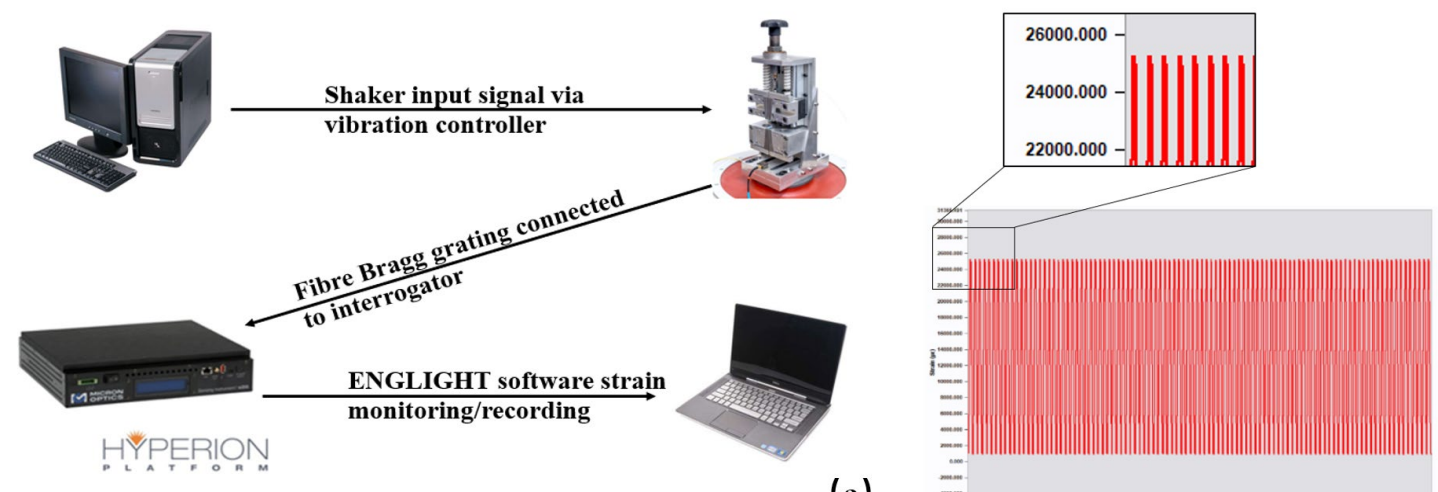

(a)

(b)

Figure 2. (a) Complete fatigue testing experimental setup. (b) An example time history of the strain response to directly monitor strain in fibre.

A total of 160 trans-jacket FBG sensors were fatigue tested. The optical fibres were supplied by OFS and FibreCore to span a range of different fibre sizes and dopant levels, as shown in Table 1. The silica fibre core was doped with germanium to increase refractive index compared to the fibre cladding and to increase photo-sensitivity.

Table 1. Optical fibre specifications.

\begin{tabular}{|l|c|c|c|c|c|c|}
\hline \multicolumn{1}{|c|}{ Optical fibre } & $\begin{array}{c}\text { Operating } \\
\text { Wavelength } \\
(\mathrm{nm})\end{array}$ & $\begin{array}{c}\text { Mode field } \\
\text { diameter, } \\
\text { nominal }(\mu \mathrm{m})\end{array}$ & $\begin{array}{c}\text { Core } \\
\text { diameter } \\
(\mu \mathrm{m})\end{array}$ & $\begin{array}{c}\text { Clad } \\
\text { diameter } \\
(\mu \mathrm{m})\end{array}$ & $\begin{array}{c}\text { Numerical } \\
\text { aperture, } \\
\text { nominal }\end{array}$ & $\begin{array}{c}\text { Dopant } \\
(\mathrm{mol} \%)\end{array}$ \\
\hline $\begin{array}{l}\text { OFS: BF06160-02 } \\
\text { ClearLite POLY } \\
(4.6 / 125,0.21 \mathrm{NA})\end{array}$ & $1310-1550$ & $\begin{array}{c}5.8 @ \\
1550 \mathrm{~nm}\end{array}$ & 4.6 & $125 \pm 2$ & 0.21 & 10 \\
\hline $\begin{array}{l}\text { OFS: BF04446 } \\
\text { ClearLite POLY } \\
(8.4 / 125,0.11 \mathrm{NA})\end{array}$ & $1310-1550$ & $\begin{array}{c}10.5 @ \\
1550 \mathrm{~nm}\end{array}$ & 8.4 & $125 \pm 2$ & 0.11 & 3 \\
\hline $\begin{array}{l}\text { Fibercore: SM1500 } \\
(4.2 \backslash 80) P\end{array}$ & $1520-1650$ & $\begin{array}{c}4.0-4.5 @ \\
1550 \mathrm{~nm}\end{array}$ & 4.2 & 80 & $0.29-0.31$ & 20 \\
\hline $\begin{array}{l}\text { Fibercore: SM1500 } \\
(9 \backslash 125) P\end{array}$ & $1550-1650$ & $\begin{array}{c}8.5-9.9 @ \\
1550 \mathrm{~nm}\end{array}$ & 9 & 125.1 & $0.13-0.15$ & 3 \\
\hline
\end{tabular}

80 gratings of each type (type I \& type II) were tested. The sensors were carefully fabricated under contract via a research agreement with an academic provider. Half of the fibres were deuterium loaded. The introduction of deuterium (D loading) can significantly reduce the energy required to inscribe a fibre grating using an IR laser, and hydrogen or deuterium loading of bulk doped glasses can significantly increase the femtosecond IR-induced index change [22].

Preliminary tests showed that type II gratings fractured at approximately $17,000 \mu \varepsilon$, while type I gratings could withstand extended cycling at strains up to $36,000 \mu \varepsilon$ (maximum range of the loading apparatus) without failure. These results informed the development of loading 
sequences used in the systematic testing of the fibres, which are shown in Tables 2 and 3 , corresponding to the type I and type II gratings respectively.

Table 2. Incremental loading sequence for type I gratings.

\begin{tabular}{|l|c|c|c|c|}
\hline Peak Strain $(\mu \varepsilon)$ & 30000 & 32000 & 34000 & 36000 \\
\hline Loading cycles (million) & 0.5 & 0.5 & 0.5 & 0.5 \\
\hline
\end{tabular}

Table 3. Incremental loading sequence for type II gratings.

\begin{tabular}{|l|c|c|c|c|}
\hline Peak Strain $(\mu \varepsilon)$ & 12000 & 14000 & 16000 & 18000 \\
\hline Loading cycles (million) & 0.5 & 0.5 & 0.5 & 0.5 \\
\hline
\end{tabular}

\section{Results and Discussion}

Table 4 shows fatigue performance results corresponding to the type I gratings. All of the type I gratings survived the full loading sequence listed in Table 2, i.e. a maximum load of $36,000 \mu \varepsilon$ and an aggregate 2 million loading cycles. These results suggest that type I gratings are suitable for applications involving severe mechanical loading, well beyond what would be experienced in a typical aerospace strain monitoring application.

Table 4. Fatigue test results for type I gratings.

\begin{tabular}{|l|c|c|c|}
\hline \multicolumn{1}{|c|}{ FBG sensors } & $\begin{array}{c}\text { Deuterium } \\
\text { loaded }\end{array}$ & $\begin{array}{c}\text { Specimens } \\
\text { tested }\end{array}$ & Fatigue results \\
\hline $\begin{array}{l}\text { OFS:BF04446, ClearLite } \\
\text { POLY (8.4/125,0.11NA) }\end{array}$ & Y\&N & 30 & $\begin{array}{c}30000-36000 \mu \varepsilon, 2 \text { million cycles } \\
\text { of loading survived }\end{array}$ \\
\hline $\begin{array}{l}\text { OFS: BF06160-02, ClearLite } \\
\text { POLY (4.6/125, 0.21NA) }\end{array}$ & Y\&N & 10 & $\begin{array}{c}30000-36000 \mu \varepsilon, 2 \text { million cycles } \\
\text { of loading survived }\end{array}$ \\
\hline FibreCore: SM1500(4.2/80) P & Y\&N & 30 & $\begin{array}{c}30000-36000 \mu \varepsilon, 2 \text { million cycles } \\
\text { of loading survived }\end{array}$ \\
\hline Fibercore: SM1500 (9/125) P & Y\&N & 10 & $\begin{array}{c}30000-36000 \mu \varepsilon, 2 \text { million cycles } \\
\text { of loading survived }\end{array}$ \\
\hline
\end{tabular}

Table 5 shows the fatigue performance results corresponding to the type II gratings. The mean fatigue failure strain for each category of type II grating was approximately $17,000 \mu \varepsilon$.

Although the failure strains of the type II gratings were significantly lower compared to that of the type I gratings, they far exceed the operational strains in most practical applications. The results were also considered against the reported fatigue performance of FSGs. The type II gratings tested in the present work significantly outperform the specialty fatigue resistant (MSeries) FSGs, which reportedly are capable of withstanding only one million loading cycles at a strain amplitude of $2500 \mu \varepsilon$ [18].

Figure 5 depicts schematically the fatigue failure locations recorded for the type II gratings (marked by a cross). As shown, all of the fractures occurred within or adjacent to the $5 \mathrm{~mm}$ sensor grating. A comparison of the pre- and post-fatigue reflection spectra (Fig. 5, top-left) shows a significant change in reflectivity in response to a fracture located within the grating. In contrast, when the fracture occurred outside of the grating there was relatively little change in reflection spectrum (Fig. 5, right), which is expected since the grating remains intact. Similar behaviour was witnessed for the type I gratings, as illustrated in Figure 6. 
Table 5. Fatigue results for type II gratings.

\begin{tabular}{|l|c|c|c|c|}
\hline Fibre tested & $\begin{array}{c}\text { Deuterium } \\
\text { loaded }\end{array}$ & $\begin{array}{c}\text { Samples } \\
\text { tested }\end{array}$ & $\begin{array}{c}\text { Mean failure } \\
\text { strain }(\mu \varepsilon)\end{array}$ & $\begin{array}{c}95 \% \text { confidence } \\
\text { interval }\end{array}$ \\
\hline $\begin{array}{l}\text { OFS: BF06160-02, ClearLite } \\
\text { POLY (4.6/125 0.21NA) }\end{array}$ & $\mathrm{Y}$ & 15 & 17600 & \pm 324 \\
\hline $\begin{array}{l}\text { OFS: BF06160-02, ClearLite } \\
\text { POLY (4.6/125 0.21NA) }\end{array}$ & $\mathrm{N}$ & 15 & 17900 & \pm 400 \\
\hline $\begin{array}{l}\text { OFS: BF04446, ClearLite } \\
\text { POLY (8.4/125 0.11NA) }\end{array}$ & $\mathrm{Y}$ & 5 & 17600 & \pm 445 \\
\hline $\begin{array}{l}\text { OFS: BF04446, ClearLite } \\
\text { POLY (8.4/125 0.11NA) }\end{array}$ & $\mathrm{N}$ & 5 & 16200 & \pm 500 \\
\hline Fibercore: SM1500 (4.2/80) P & $\mathrm{Y}$ & 5 & 17300 & \pm 588 \\
\hline Fibercore: SM1500 (4.2/80) P & $\mathrm{N}$ & 5 & 18100 & \pm 480 \\
\hline FibreCore: SM1500(9/125) P & $\mathrm{Y}$ & 15 & 17100 & \pm 473 \\
\hline FibreCore: SM1500(9/125) P & $\mathrm{N}$ & 15 & 16400 & \pm 643 \\
\hline
\end{tabular}

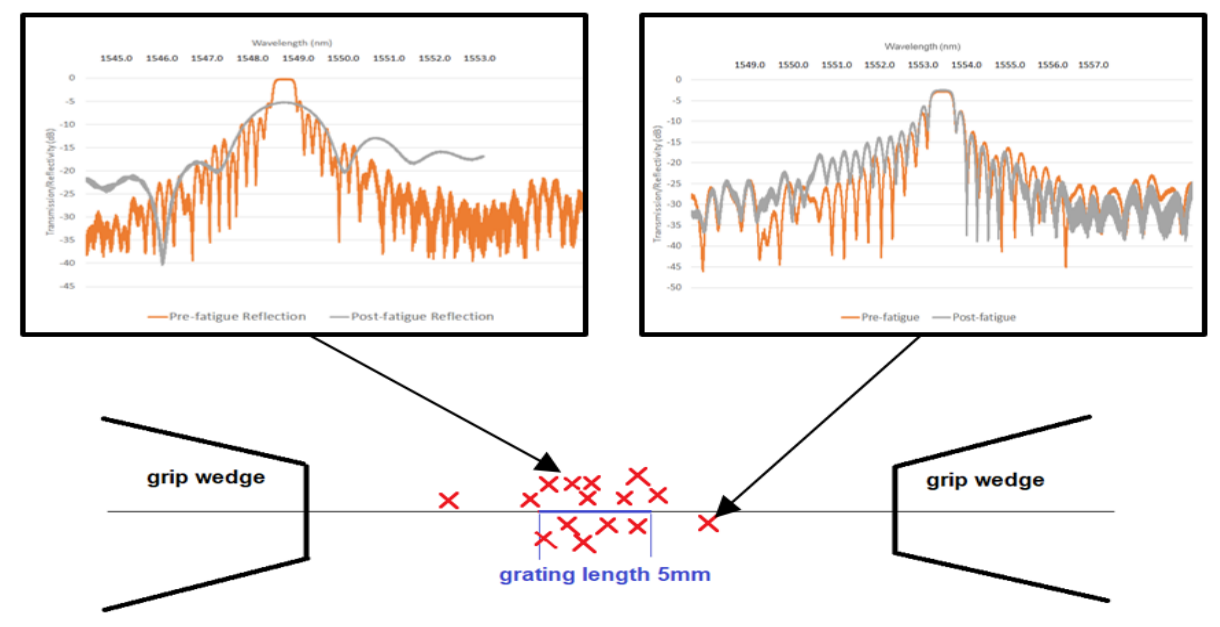

$\times$ Relative failure locations

Figure 5. Summary of failure locations schematically represented for one sample set of type II gratings.

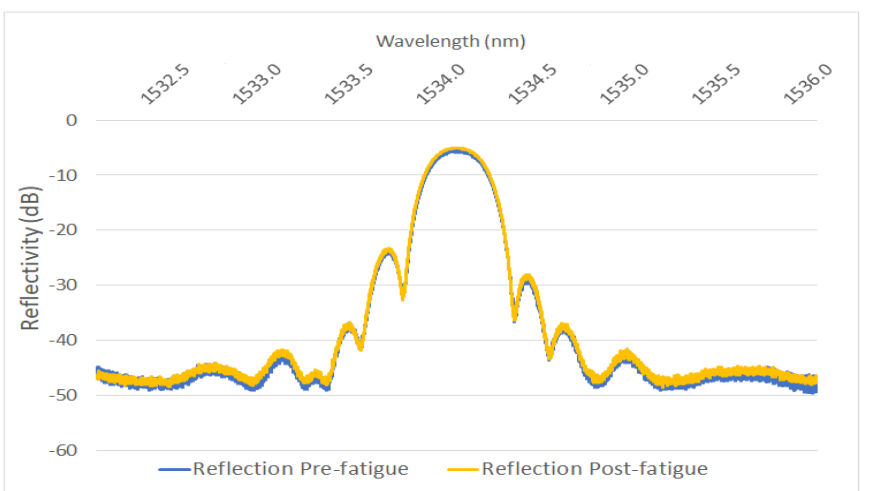

Figure 6. Pre- and post-fatigue reflection spectra of a type I grating. 


\section{Conclusions}

This paper has reported on the durability and reliability of Bragg gratings with different fibre geometries, dopants, and photo-sensitising approaches to compare the overall fatigue performance of type I and type II trans-jacket FBG sensors. The fatigue performance of these sensors was assessed using a custom designed electro-dynamically actuated loading assembly. It was concluded that type I trans-jacket gratings have a significantly higher fatigue life compared to type II gratings for the same fatigue loading regime. Despite the lower fatigue life, type II trans-jacket gratings are found to perform significantly better than conventional electrical foil gauges. Therefore, trans-jacket gratings have strong potential for application as dense sensing arrays in harsh operational environments in defence and aerospace industries.

The results also illustrated that photo-sensitising, glass dopant and fibre geometry had no discernable impact on the mechanical performance of trans-jacket FBGs. The next stage of this research will focus on developing and testing reliable broad-area fatigue resistant attachment techniques for this new class of sensor.

\section{Funding}

This research is funded by US Navy Office of Naval Research (N62909-18-1-2063). The financial supported provided by the Office of Naval Research is gratefully acknowledged.

\section{Conflict of Interest}

The author declares that there is no conflict of interest regarding the publication of this paper.

\section{References}

[1] G. Meltz, W. W. Morey, and W. H. Glenn, "Formation of Bragg gratings in optical fibers by a transverse holographic method," Opt. Lett., 1989, doi: 10.1364/ol.14.000823.

https://doi.org/10.1364/OL.14.000823

[2] R. Kashyap, Fiber Bragg Gratings. 2010. https://doi.org/10.1016/B978-0-12-372579$0.00004-1$

[3] A. D. Kersey et al., "Fiber grating sensors," J. Light. Technol., vol. 15, no. 8, pp. 14421462, 1997. https://doi.org/10.1109/50.618377

[4] Y. J. Rao, “In-fibre Bragg grating sensors," Meas. Sci. Technol., vol. 8, no. 4, pp. 355-375, 1997. https://doi.org/10.1088/0957-0233/8/4/002

[5] M. Majumder, T. K. Gangopadhyay, A. K. Chakraborty, K. Dasgupta, and D. K. Bhattacharya, "Fibre Bragg gratings in structural health monitoring-Present status and applications," Sensors and Actuators, A: Physical. 2008. https://doi.org/10.1016/j.sna.2008.04.008

[6] R. Di Sante, "Fibre optic sensors for structural health monitoring of aircraft composite structures: Recent advances and applications," Sensors (Switzerland). 2015. https://doi.org/10.3390/s150818666

[7] R. Ramly, W. Kuntjoro, and M. K. A. Rahman, "Using embedded fiber bragg grating (FBG) sensors in smart aircraft structure materials," 2012. https://doi.org/10.1016/j.proeng.2012.07.218

[8] K. C. Chuang and C. C. Ma, "Tracking control of a multilayer piezoelectric actuator using a fiber bragg grating displacement sensor system," IEEE Trans. Ultrason. Ferroelectr. Freq. Control, 2009. https://doi.org/10.1109/TUFFC.2009.1287 
[9] M. W. Rothhardt, C. Chojetzki, and H. R. Mueller, "High-mechanical-strength single-pulse draw tower gratings," 2004. https://doi.org/10.1117/12.567801

[10] K. O. Hill and G. Meltz, "Fiber Bragg grating technology fundamentals and overview," J. Light. Technol., 1997. https://doi.org/10.1109/50.618320

[11] H. J. Yoon and C. G. Kim, "The mechanical strength of fiber Bragg gratings under controlled UV laser conditions," Smart Mater. Struct., 2007. https://doi.org/10.1088/0964$1726 / 16 / 4 / 045$

[12] J. Ang, H. C. H. Li, I. Herszberg, M. K. Bannister, and A. P. Mouritz, "Tensile fatigue properties of fibre Bragg grating optical fibre sensors," Int. J. Fatigue, 2010. https://doi.org/10.1016/j.ijfatigue.2009.11.002

[13] M. Bernier, F. Trépanier, J. Carrier, and R. Vallée, "High mechanical strength fiber Bragg gratings made with infrared femtosecond pulses and a phase mask," Opt. Lett., vol. 39, no. 12, p. 3646, 2014. https://doi.org/10.1364/OL.39.003646

[14] J. Habel, T. Boilard, J. S. Frenière, F. Trépanier, and M. Bernier, "Femtosecond FBG written through the coating for sensing applications," Sensors (Switzerland), vol. 17, no. 11, pp. 1-12, 2017. https://doi.org/10.3390/s17112519

[15] C. W. Smelser, S. J. Mihailov, and D. Grobnic, "Formation of Type I-IR and Type II-IR gratings with an ultrafast IR laser and a phase mask," Opt. Express, 2005.

https://doi.org/10.1364/OPEX.13.005377

[16] L. Sudrie, M. Franco, B. Prade, and A. Mysyrowicz, "Study of damage in fused silica induced by ultra-short IR laser pulses," Opt. Commun., 2001. https://doi.org/10.1016/S00304018(01)01152-X

[17] C. Davis, S. Tejedor, I. Grabovac, J. Kopczyk, and T. Nuyens, "High-strain fiber bragg gratings for structural fatigue testing of military aircraft," Photonic Sensors, 2012. https://doi.org/10.1007/s13320-012-0066-3

[18]“The Fatigue Life of Electrical Strain Gauges,” HBM, 2020. https://www.hbm.com/en/8153/new-technote-fatigue-life-of-electrical-strain-gauges/ (accessed Oct. 27, 2020).

[19] J. C. Middleton, "Strain gauge performance during fatigue testing," Strain, vol. 22, no. 3, pp. 135-140, 1986. https://doi.org/10.1111/j.1475-1305.1986.tb00608.x

[20] N. Zhang, C. Davis, W. K. Chiu, T. Boilard, and M. Bernier, "Fatigue performance of type I fibre bragg grating strain sensors," Sensors (Switzerland), vol. 19, no. 16. 2019. https://doi.org/10.3390/s19163524

[21] S. R. Abdullina and A. A. Vlasov, "Suppression of side lobes in the fiber Bragg grating reflection spectrum," Optoelectron. Instrum. Data Process., 2014.

https://doi.org/10.3103/S8756699014010105

[22] C. W. Smelser, S. J. Mihailov, and D. Grobnic, "Hydrogen loading for fiber grating writing with a femtosecond laser and a phase mask," Opt. Lett., vol. 29, no. 18, p. 2127, 2004.

https://doi.org/10.1364/OL.29.002127 